\title{
Hyperlipidaemia - a Risk Factor for Femoral Head Osteonecrosis (Legg-Calvè- Perthes-Like Disease) in Children With AIDS: Case Report
}

Paula Aguiar de Aragão, Renata B. P. Lemos,

Maria Zilda Aquino, and Heloísa Helena Souza Marques

\author{
Department of Infectious Diseases, Children's \\ Institute, Clinical Hospital, Faculty of \\ Medicine of the University of São Paulo, SP, \\ Brazil
}

\begin{abstract}
Although treatment of children infected with HIV with protease inhibitors has improved the survival of these patients, various adverse side effects have been reported, including metabolic abnormalities, such as hyperlipidaemia. We describe a case of hip osteonecrosis in an adolescent with AIDS who was being treated with protease inhibitors. There is a possible relation with hyperlipidemia. F.M.G., white, 11 years old, AIDS A2, started to receive AZT and DDI when he was 7 years old. In April 1999, the patient had a significant increase in viral load and so the antiretroviral therapy was switched to $\mathrm{d} 4 \mathrm{~T}, 3 \mathrm{TC}$ and Ritonavir. Triglyceride plasma levels reached $460 \mathrm{mg} / \mathrm{dl}$ after this switch and were always above the reference value. In December 1999, the patient complained of pain in the right hip. On physical examination, he had limited movement of this joint. Magnetic resonance imaging of the right hip showed flattening, deformity and fragmentation of the femoral head, compatible with osteonecrosis. Few cases of femoral head osteonecrosis have been associated with HIV infection, in the absence of the classic risk factors for osteonecrosis. Metabolic risk factors include hypertriglyceridaemia. The immunological disorders that occur in the HIV infection may predispose the patient to avascular osteonecrosis and metabolic disorders, particularly hypertriglyceridemia, while the use of protease inhibitors, may be considered an additional risk factor for osteonecrosis. Given the importance of premature diagnosis and to avoid complications of osteonecrosis, we recommend evaluation of musculoskeletal symptoms in children receiving protease inhibitors.
\end{abstract}

Key Words: Hyperlipidaemia, femoral head osteonecrosis, children, AIDS.

Although the use of protease inhibitors for the treatment of children infected with human immunodeficiency virus (HIV) has improved the survival and quality of life of these patients, adverse side effects have been reported. Among them, metabolic abnormalities, including hyperlipidemia (hypertriglyceridemia and especiallyhypercholesterolemia), have been described [1]. An association between hypertriglyceridemia and osteonecrosis in patients with AIDS has been suggested [4].

Received on17 February 2002; revised 04 June 2002.

Address for correspondence: Dr. Paula Aguiar de Aragão. Rua Visconde de Inhaúma, 535, apt. 32, Vila da Saúde, Zip Code: 04145-030, São Paulo, SP, Brazil. Phone: (55 11) 5594-0314.

The Brazilian Journal of Infectious Diseases 2002;6(3):142-145 (C) 2002 by The Brazilian Journal of Infectious Diseases and Contexto Publishing. All rights reserved. $1413-8670$
In children, osteonecrosis of the hip may be LeggCalvè-Perthes disease. This is a self-limiting disease, with a chronic course and unknown etiology, attacking especially pre-school age boys and can result in member shortening and deformity of the femoral head [2,6-10]. Few cases of femoral head osteonecrosis have been described during HIV infection [1] and none of them in pediatric patients. The objective of this report is to describe a case of hip osteonecrosis in an adolescent with AIDS, who was receiving protease inhibitor and alert to a possible association with hyperlipidemia.

\section{Case Report}

F.M.G., white, 11 years old, vertically transmitted AIDS A2 (CDC), treated in our hospital since January 
1996, when he was 7 years old. He was asymptomatic at this time. In his admission exams, the $\mathrm{CD}_{4}$ lymphocyte count was $391 \mathrm{cell} / \mathrm{mm}^{3}$. In August 1996, the patient started to receive AZT and DDI, and during the next year he had no clinical symptoms, a $\mathrm{CD}_{4}$ lymphocyte count of $454 \mathrm{cell} / \mathrm{mm}^{3}$ and a viral load of 2,050 copies/ml. In August 1997, the patient had pain in the right shoulder but the x-ray of the region was normal and there was spontaneous remission in a few days. From May to October 1998, he had chronic parothiditis and an increase in viral load to 13,100 copies/ml, so ddI was changed to 3TC in June 1998, with good laboratory and clinical responses. In April 1999 , the patient had an large increase in viral load $(24,719$ copies/ml $)$ and then the antiretroviral therapy was switched to d4T, 3TC and Ritonavir. The viral load was reduced to 790 copies/ml (May 1999), the lymphocyte count was $481 \mathrm{cell} / \mathrm{mm}^{3}$ and the patient was asymptomatic. The plasma triglyceride levels reached $460 \mathrm{mg} / \mathrm{dl}$ (reference value: $100 \mathrm{mg} / \mathrm{dl}$ ) after this switch and were always above the reference value. On December 6, 1999, the patient came to the hospital with pain in the right hip, beginning 2 or 3 weeks before, progressive and worsening during the last four days. The patient reported constant pain and there was no improvement with common analgesics and rest. Sporadically, he had pain in the legs after strenuous effort (such as playing soccer), but always of low intensity. He had no fever or other signs and symptoms. On physical examination, he had difficulty in walking, and did so with an antalgic limp, diffuse pain in the right hip, pain on flexion of the right knee and limited motion, especially with external rotation of the hip. There were no signs of inflammation. The ultrasound exam made the same day showed flattening of the femoral epiphysis with ossification nucleous fragmentation and no joint effusion. The x-ray of the hip showed asymmetric ossification nucleous and flattening and fragmentation of the right hip.

On December 7, 1999, he was hospitalized to be treated with analgesics and to program the orthopedic treatment. Other exams included: chest roentgenogram showing a nonspecific bilateral infiltrate, PPD negative, alkaline phosphatase 325UI/1 (38-126), thyroid- stimulating hormone $7.02 \mathrm{um} / \mathrm{ml}(0.35-5.50)$ with triiodothyromine (T3) and thyroxin (T4) at normal levels. The phosphorus, ionized and urinary calcium and phosphorus levels were also normal. The magnetic resonance imaging (MRI) of the right hip showed flattening, deformity and fragmentation of the femoral head, with decreased uptake on T1 and T2, compatible with osteonecrosis; there was joint effusion and the medial part of the epiphysis was not involved. The MRI of the left hip showed a small lesion $(1.0 \times 0.5 \mathrm{~cm})$ on the femoral epiphysis, compatible with a necrotic fragment. The patient improved after analgesics that included paracetamol and tramadol and was discharged on December 10, using crutches. During ambulatorial care, the pain and the mobility of the right hip improved and the crutches were suspended. The control x-ray, on December 28 showed bilateral deformity of the femoral heads, especially on the right side, where it was subluxated, and the right acetabulum was deformed. Currently, the patient is returning to his habitual activities, including school.

\section{Discussion}

Recently, hyperlipidaemia has been indicated as another risk factor for osteonecrosis [1]. However, few cases of femoral head osteonecrosis have been associated with HIV infection, in the absence of the other classic risk factors to osteonecrosis [1].

Aseptic necrosis is probably an ischemic disease (with bone blood supply interruption sufficient to cause tissue damage) $[3,4]$. In children, when occurs on the femoral head, it is Legg-Calvè-Perthes disease. This is a self-limiting disease of the hip, with unknown etiology and results in variable degrees of epiphyseal deformity and restricted articular motion [6-10]. It has a chronic course and osteoarthritic changes can develop in adult life $[6,10]$. It affects both sexes, with a male predominance of 4 to $5: 1$, generally between the ages of 3 and 10 and it is rare in blacks $[2,6,9]$. Bone age is invariably delayed by 1 to 3 years compared to chronologic age $[6,8]$. Its pathology involves loss of blood supply to the femoral epiphysis with avascular 
Figure 1. Ossification nucleous asymmetric and flattening and fragmentation of the right hip

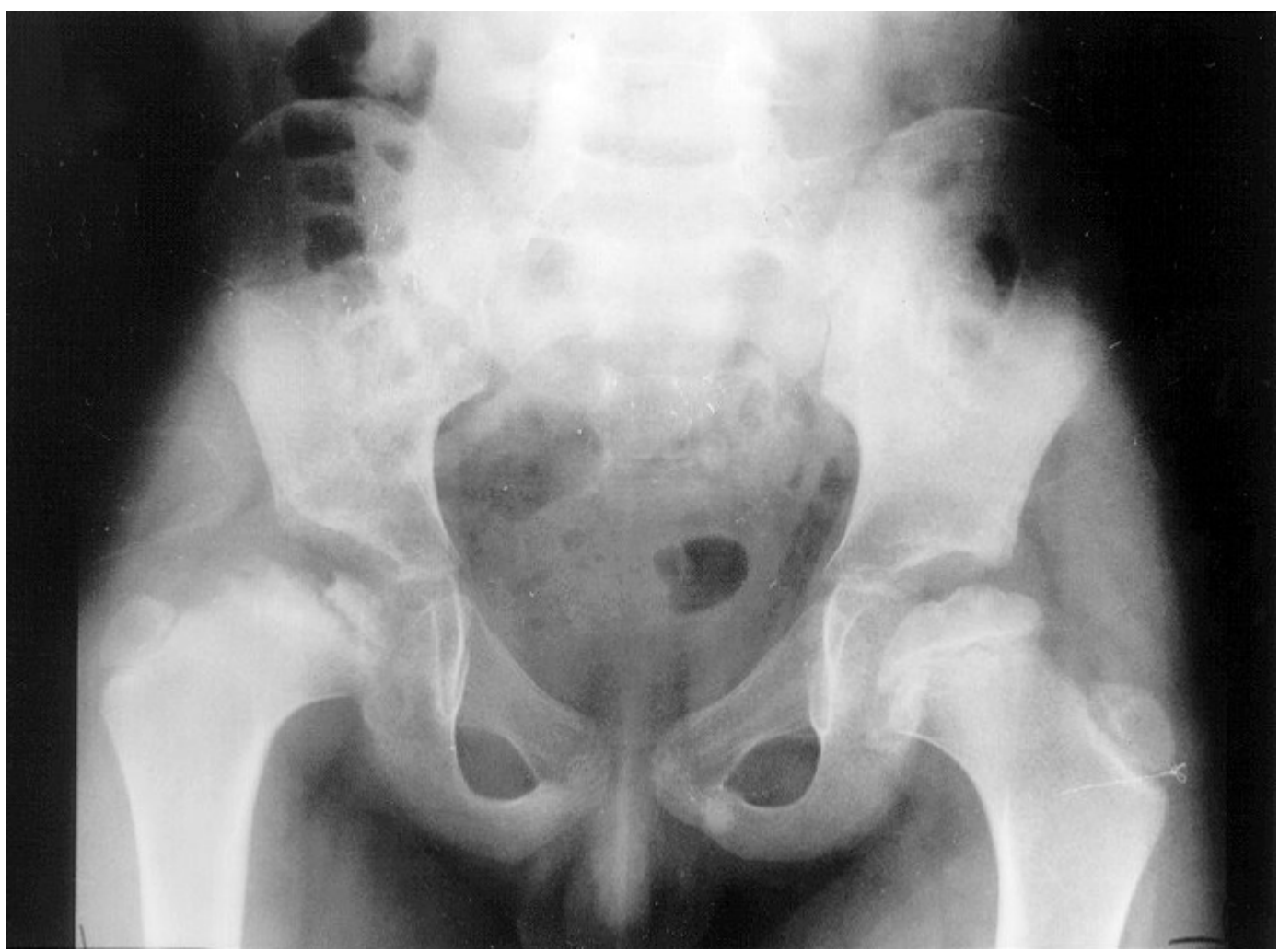

necrosis, followed by resorption of the dead bone and replacement with newly formed immature bone [2,610]. It has characteristic $x$-ray findings and it has a well known classification into four groups (Caterral, 1972) based on the extent of epiphyseal involvement and its prognosis $[2,6,7,9]$.

Although there is no identifying risk factor for osteonecrosis in most patients, a lot of precipitating factors have been identified and include: local trauma (most commonly intertrochanteric hip fracture and slipped femoral epiphysis), barotrauma, chronic alcoholism, coagulopathies, sickle-cell anaemia and other hemoglobinopathies, systemic erythematous lupus, leukemia, gout, pancreatitis, tuberculosis, haemodialysis, steroid therapy and Gaucher's disease [1-4]. Metabolic risk factors include hypertriglyceridemia and anticardiolipin antibodies [4]. Multiple pathological mechanisms have been suggested, including mechanical vascular interruption, thrombosis and embolism, injury to blood vessel walls, elevated internal marrow pressure, immune complex disease and anticardiolipin antibodies [2-4,9]. The hip joints are, according to the literature, the commonest site of avascular necrosis in HIV infection [5]. Its occurrence early in the course of HIV infection suggests that it could be a consequence of metabolic or immunological changes, rather than the consequence of infectious complications of HIV or AIDS [3]. 
This is the first case of osteonecrosis and AIDS in a child and alerts to a possible relationship with hypertriglyceridemia induced by protease inhibitors, as no specific risk factor was found. Osteonecrosis may have occurred by the disease's evolution itself or by a simple coincidence, as the patient had short stature and bone age delayed 5 years compared to the chronological age. However, since there was a temporal relation between hypertriglyceridemia (4 times the normal levels) and the occurrence of the first symptoms, we consider possible and probable the correlation with osteonecrosis.

In conclusion, the immunological disorders that occur early in the HIV infection may predispose to avascular osteonecrosis $[3,4]$ and the metabolic disorders, particularly hypertriglyceridemia, from the use of protease inhibitors, may be considered another risk factor for the development of osteonecrosis [1]. Given the importance of early diagnosis and given the complications of osteonecrosis, such as deformity and shortening of members, we recommend evaluation of musculoskeletal symptoms in children receiving protease inhibitors.

\section{References}

1. Meyer D., Behrens G., Schmidt R.E., Stoll M. Osteonecrosis of the femoral head in patients receiving HIV protease inhibitors. AIDS 1999;13(9):1147-8.

2. Resnick D., Nuwayama G. In: Diagnosis of Bone and Joint Disorders, second edition, pg. 3290-304, 1988.

3. Rademaker J., Dobro J.S., Solomon G. Osteonecrosis and Human Immunodeficiency Virus Infection The Journal of Rheumatology 1997;24(3):601-4.

4. Chevalier X., Larget-Piet B., Hernigou P., Gherardi R. Avascular necrosis of the femoral head in HIV-infected patients. The Journal of Bone and Joint Surgery 1993;75-B: 160 .

5. Gerster J.C., Rossetti G. Aseptic avascular osteonecrosis mimicking arthritis in HIV infection. The Journal of Rheumatology 1998;25(3): 604-5.

6. Turek S.L. Coxa plana in: Orthopedics - Principles \& Their Application, fourth edition vol. 2: 1219-37, 1984.

7. Duthie R.B., Bentley G. Legg-Calvè-Perthes disease in: Mercer's Orthopedic Surgery, eighth edition, pg. 313322, 1983.
8. Behrman R.E, Kliegman R.M., Nelson W.E. Legg-CalvèPerthes disease in: Textbook of Pediatrics, fourteenth edition, W.B. Saunders Company, 1992.

9. Bruschini S. Doença de Legg-Calvè-Perthes (Osteocondrite da cabeça do fêmur) in: Ortopedia Pediátrica, 1993.

10. Adams J.C., Hamblen D.L. Doença de Perthes in: Manual de Ortopedia, 14 ${ }^{\text {th }}$ edition, pg. 338-47, 1994. 